\title{
A Global Sensitivity Analysis Framework for Hybrid Simulation
}

\author{
G. Abbiati*1, S. Marelli², N. Tsokanas², B. Sudret ${ }^{2}$, and B. Stojadinovic ${ }^{2}$ \\ ${ }^{1}$ Department of Civil and Architectural Engineering, Aarhus University, Denmark \\ ${ }^{2}$ Department of Civil, Environmental and Geomatic Engineering, ETH Zurich, Switzerland
}

Draft submitted to engrXiv

May 5, 2020

\begin{abstract}
Hybrid Simulation is a dynamic response simulation paradigm that merges physical experiments and computational models into a hybrid model. In earthquake engineering, it is used to investigate the response of structures to earthquake excitation. In the context of response to extreme loads, the structure, its boundary conditions, damping, and the ground motion excitation itself are all subjected to large parameter variability. However, in current seismic response testing practice, Hybrid Simulation campaigns rely on a few prototype structures with fixed parameters subjected to one or two ground motions of different intensity. While this approach effectively reveals structural weaknesses, it does not reveal the sensitivity of structure's response. This thus far missing information could support the planning of further experiments as well as drive modeling choices in subsequent analysis and evaluation phases of the structural design process.

This paper describes a Global Sensitivity Analysis framework for Hybrid Simulation. This framework, based on Sobol' sensitivity indices, is used to quantify the sensitivity of the response of a structure tested using the Hybrid Simulation approach due to the variability of the prototype structure and the excitation parameters. Polynomial Chaos Expansion is used to surrogate the hybrid model response. Thereafter, Sobol' sensitivity indices are obtained as a by-product of polynomial coefficients, entailing a reduced number of Hybrid Simulations compared to a crude Monte Carlo approach. An experimental verification example highlights the excellent performance of Polynomial Chaos Expansion surrogates in terms of stable estimates of Sobol' sensitivity indices in the presence of noise caused by random experimental errors.
\end{abstract}

Keywords: Hybrid simulation ; global sensitivity analysis ; Sobol' indices ; surrogate modeling ; polynomial chaos expansion.

\section{Introduction}

Background and motivation Hybrid Simulation (HS) is a dynamic simulation paradigm that merges physical experiments and computational models into a hybrid model. In mechanical and electrical engineering, such an approach is known as hardware-in-the-loop simulation. In earthquake engineering, hybrid simulation is used to investigate the behavior and eventual failure of a structure in response to extreme earthquake excitation. In detail, HS is conducted using a hybrid model, which combines physical and numerical substructures (NS and PS, respectively) that interact with each other in a real-time control loop, to simulate the time history response of a prototype structure subjected to a realistic excitation. The PS is tested in the laboratory using servo-controlled actuators equipped with force and displacement transducers. The NS is numerically simulated to avoid testing of those substructures that can be reliably replaced by numerical models (e.g., masses or components whose response remains in an easy-to-simulate range) or would exceed the testing capacities of the experimental facility. A real-time software ensures interface force balance and displacement compatibility between NS and PS by solving the equation of motion of the prototype structure online, that is, while structural testing is performed. As a result, HS reproduces the time history response of the prototype structure subjected to a realistic excitation. When the PS response is rate-independent, it is good practice to perform HS with an extended time scale from 50 to 200 times slower than real-time in order to improve control accuracy. Time scaling and testing of geometrically scaled substructures also drastically reduce the costs of HS compared

${ }^{*}$ Corresponding Author: Dr. Giuseppe Abbiati, abbiati@cae.au.dk, ORCID: 0000-0002-5048-8505. 
to full-scale experimentation. The seminal report by Schellenberg and Mahin [1] provides a comprehensive overview of state-of-art HS methods and algorithms.

HS is suitable either to investigate the behavior of a prototype structure at the system level, where PSs replace components lacking validated numerical models, or to observe the component-level behavior of PSs exposed to a realistic excitation, which includes dynamic interaction with possibly multiple NSs. The overarching goal is to support the validation and calibration of computer models [2]. A system-level focus is pursued in earthquake engineering, where HS is preferred to shake table experiments for testing large structures such as bridges (e.g., [3]) and buildings (e.g., [4]). Similarly, testing of floating platforms with a sizeable scaling factor in hydrodynamic laboratories is challenging due to the extent of mooring line footprints, which can range from two to four times the water depth. For this reason, HS has been recently proposed to truncate mooring lines whose missing parts are simulated as NSs [5]. A component-level focus is pursued in fire engineering, where hybrid fire testing, the adaptation of HS to fire loading, originated in response to limitations of standard fire tests [6]. The latter consists of exposing a single structural component to a predefined time-temperature loading curve inside a furnace and indeed does not account for the redistribution of internal forces caused, for example, by thermal expansion or partial collapse. For a similar motivation, Franza and Marshall [7] developed an HS platform to study soil-structure interaction problems where a numerical model of a building frame (i.e., the NS) is used to mimic a realistic structural loading exerted on the physical centrifuge model of a tunnel-soil-foundation system (i.e., the PS). The use of HS in other engineering and science disciplines is growing (e.g. [8], [9]).

In earthquake engineering, which is the main field of expertise of some of the authors, boundary conditions, damping, and ground motion are all subjected to large parameter variability [10]. However, in the current experimental earthquake engineering practice, HS campaigns rely on a few prototype structures with fixed parameters subjected to one or two ground motions of different intensity. This testing procedure efficiently identifies the weaknesses of the tested prototype that could lead to the collapse of real structures in earthquakes. However, such a truncated procedure is indeed not adequate to estimate the structural sensitivity of the prototype structure response, i.e. quantifying the fraction of the variability of a generic response quantity of interest (QoI) due to the variability of each of the input parameters. This thus far missing information could support the planning of further experiments as well as drive modeling choices in subsequent analysis and evaluation phases of the structural design process.

Scope This paper describes a Global Sensitivity Analysis (GSA) framework for Hybrid Simulation that is intended to quantify the fraction of the variability of a generic response QoI of the prototype structure, evaluated via HS, due to the variability of a set of input variables that provide a suitable parametrization of the prototype structure and the excitation. Among the many GSA options, Sobol' sensitivity indices, a class of variancedecomposition-based methods [11], were selected. Methods of this class rely upon the decomposition of the variance of a QoI as a sum of contributions of each input variable taken singularly or in combination with others. Unlike regression-based methods, variance-based methods do not require any kind of linearity or monotonicity of the functional relationship between input variables and QoIs.

An efficient way to compute Sobol' indices, originally introduced by Sudret [12, 13], is by post-processing the coefficients of a Polynomial Chaos Expansion (PCE) of the QoI. PCE is a spectral decomposition method that projects the model response onto a functional basis of orthonormal multivariate polynomials in the input variables [14, 15]. Once the PCE representation is available, Sobol' indices are readily available by simply postprocessing polynomial coefficients with no additional computational cost. The strategies for the calculation of the PCE can be broadly classified into two approaches: i) intrusive i.e., schemes that require the knowledge and adaptation of the governing equations of the model; and ii) non-intrusive i.e., schemes that (repeatedly) utilize the model to compute an outcome without modifying the model itself, which is considered as a black-box. HS and its PSs, and experimental testing in general, imply a non-intrusive PCE strategy. Hence, in this paper, the sparse adaptive least-square minimization based on Least Angle Regression (LAR) [16] was adopted. The proposed GSA framework for HS is illustrated using a case study based on a 3-degrees-of-freedom (3-DoFs) prototype structure excited by earthquake ground motions. HSs are performed using a 3-DoFs HS test rig installed at the Structural Laboratory of ETH Zurich. The GSA of the hybrid model response is performed for two response QoIs against four input variables, which parametrize both the NS and the excitation.

While to the best knowledge of the authors this is a pioneering application of GSA to HS, it is important to remark that the goal of this study is not to quantify the generic sensitivity of the model response to uncertain inputs as in the context of uncertainty quantification using Sobol' sensitivity analysis. Instead, the proposed procedure aims to identify the primary drivers of the hybrid model response, that is, to better understand the inner workings of the hybrid model itself and thus inform the design of a HS campaign. For a discussion on 
some recent applications of GSA outside structural engineering see e.g., [17, 18, 19]. The earthquake engineering validation case study was selected because this is the main applied field of expertise of some of the authors and because of the available test setups. However, the proposed procedure treats the hybrid model as a black-box, and is thus applicable to any dynamic system response investigated via HS.

The paper is organized as follows. Section 2 describes the GSA framework based on PCE. Section 3 describes the case study, including a detailed description of the HS setup and the HSs. Section 4 presents the results of the GSA of the prototype structure response. Finally, conclusions and future outlook are given.

\section{Global Sensitivity Analysis framework}

Let us denote with $\mathbf{X}=\left\{X_{1}, \ldots, X_{M}\right\}$ an $M$-dimensional vector of input variables to parametrize the NSs, the PSs and the excitation (or a subset of these) of the hybrid model of the prototype structure, and with $Y$ a generic response QoI of the prototype structure evaluated via HS of the response of the hybrid model. A mapping $\mathcal{M}$ between input variables and a single QoI reads,

$$
\mathbf{X} \in \mathcal{D}_{\mathbf{X}} \subset \mathbb{R}^{M} \mapsto Y=\mathcal{M}(\mathbf{X}) \subset \mathbb{R}
$$

It can be argued that the inherent variability of the response of different samples corresponding to nominally identical PSs of the hybrid model, as well as measurement noise during HS, which both propagate through to the hybrid model response QoI, requires a stochastic mapping between input variables and QoIs. However, the scope of this paper is limited to the case of PSs with almost deterministic behavior. Moreover, relevant studies testify that measurement noise of standard structural testing equipment has a negligible effect on the prototype structure response evaluated via HS [20, 21]. Therefore, $\mathcal{M}$ is assumed to be deterministic. Since our target is to estimate the structural sensitivity of the prototype structure response, which means quantifying the fraction of the variability of a generic QoI due to the variability of the input variables, all input variables in $\mathbf{X}$ are assumed to be statistically independent and uniformly distributed. The finite bounds of input variables compatible with force and displacement ranges allowed by the experimental facility are the only constraints of $\mathbf{X}$.

\subsection{Sobol'-Hoeffding decomposition and sensitivity indices}

Assume that the vector of input variables $\mathbf{X}$ has support $\mathcal{D}_{\mathbf{X}}$ and follows an independent joint probability density function (PDF) $f_{\mathbf{X}}(\mathbf{x})=\prod_{i}^{M} f_{X_{i}}\left(x_{i}\right)$ where $f_{X_{i}}$ is the marginal PDF of the $i$-th input variable. Any square-integrable mapping $Y=\mathcal{M}(\mathbf{X})$ with respect to the probability measure associated with $f_{\mathbf{X}}$, can be written as a sum of functions of increasing dimension as [22]:

$$
\mathcal{M}(\mathbf{X})=\mathcal{M}_{0}+\sum_{i=1}^{M} \mathcal{M}_{i}\left(X_{i}\right)+\sum_{1 \leq i \leq j \leq M} \mathcal{M}_{i, j}\left(X_{i}, X_{j}\right)+\ldots+\mathcal{M}_{1,2, \ldots, M}(\mathbf{X}),
$$

or equivalently:

$$
\mathcal{M}(\mathbf{X})=\mathcal{M}_{0}+\sum_{\mathbf{u} \neq \emptyset} \mathcal{M}_{\mathbf{u}}\left(\mathbf{X}_{\mathbf{u}}\right)
$$

where $\mathcal{M}_{0}$ is the mean value of $Y, \mathbf{u}=\left\{i_{1}, \ldots, i_{s}\right\} \subset\{1, \ldots, M\}$ are index sets, and $\mathbf{X}_{\mathbf{u}}$ denotes a subvector of $\mathbf{X}$ containing only the components indexed by $\mathbf{u}$. The number of summands in the above equation is $2^{M}-1$. The Sobol' decomposition is unique under the condition:

$$
\int_{\mathcal{D}_{X_{k}}} \mathcal{M}_{\mathbf{u}}\left(\mathbf{x}_{\mathbf{u}}\right) f_{X_{k}}\left(x_{k}\right) d x_{k}=0, \text { if } k \in \mathbf{u},
$$

where $\mathcal{D}_{X_{k}}$ and $f_{X_{k}}$ denote the support and marginal PDF of $X_{k}$, respectively, which leads to the orthogonality property:

$$
\mathbb{E}\left[\mathcal{M}_{\mathbf{u}}\left(\mathbf{x}_{\mathbf{u}}\right) \cdot \mathcal{M}_{\mathbf{v}}\left(\mathbf{x}_{\mathbf{v}}\right)\right]=0, \text { if } \mathbf{u} \neq \mathbf{v} .
$$

The uniqueness and orthogonality properties allow for the following decomposition of the variance $D$ of $Y$ :

$$
D=\operatorname{Var}[\mathcal{M}(\mathbf{X})]=\sum_{\mathbf{u} \neq \emptyset} \mathcal{D}_{\mathbf{u}}
$$


where $\mathcal{D}_{\mathbf{u}}$ denotes the partial variance:

$$
\mathcal{D}_{\mathbf{u}}=\operatorname{Var}\left[\mathcal{M}_{\mathbf{u}}\left(\mathbf{x}_{\mathbf{u}}\right)\right]=\mathbb{E}\left[\mathcal{M}_{\mathbf{u}}^{2}\left(\mathbf{x}_{\mathbf{u}}\right)\right]
$$

The Sobol' index $S_{\mathbf{u}}$ can be defined as the fraction of the total variance $D_{\mathbf{u}}$ that corresponds to the set of input variables indexed by $\mathbf{u}$ :

$$
S_{\mathbf{u}}=\frac{D_{\mathbf{u}}}{D}
$$

By construction, $\sum_{\mathbf{u} \neq \emptyset} S_{\mathbf{u}}=1$. First-order indices $S_{i}^{(1)}$ describe the influence of each parameters $X_{i}$ considered separately. Second-order indices $S_{i j}^{(2)}$ describe the influence from pairs of parameters $\left\{X_{i}, X_{j}\right\}$ not already accounted for by $X_{i}$ or $X_{j}$ separately. High-order indices describe combine influences from larger sets of parameters. The total sensitivity indices $S_{i}^{(\text {tot })}$ represent the total effect of an input variable $X_{i}$ accounting for its main effect and all interactions with other input variables. It follows that $S_{i}^{(\text {tot })}=1-S_{\sim i}$, where $S_{\sim i}$ is the sum of all $S_{\mathbf{u}}$ with $\mathbf{u}$ not including $i$. Sobol' indices can be evaluated by Monte Carlo simulation [11], which requires $\mathcal{O}\left(10^{3}\right)$ model evaluations for each index $S_{\mathbf{u}}$. Clearly, this is not affordable when each evaluation of $\mathcal{M}(\mathbf{x})$ entails the execution of a HS. On the other hand, when the PCE of the QoI is available, Sobol' indices can be obtained analytically at no additional cost. A concise description of PCE-based Sobol' sensitivity indices estimation is given in the following section; for further details, the reader is referred to [12, 13].

\subsection{Sobol' indices from Polynomial Chaos Expansion}

Sparse PCE [16] is a well-known surrogate modeling technique. Its close relation to Sobol' variance decomposition [12] together with good denoising performance [23] make sparse PCE ideal to surrogate the prototype structure response evaluated via HS. PCE relies on the decomposition of a random model response QoI $Y=\mathcal{M}(\mathbf{X})$ as a linear superposition of non-linear functions as follows:

$$
\widehat{Y}=\mathcal{M}^{\mathrm{PCE}}(\mathbf{X})=\sum_{\boldsymbol{\alpha} \in \mathcal{A}} y_{\boldsymbol{\alpha}} \Psi_{\boldsymbol{\alpha}}(\mathbf{X}),
$$

where $\left\{\Psi_{\alpha}, \boldsymbol{\alpha} \in \mathcal{A}\right\}$ is a set of multivariate polynomials that are orthogonal with respect to the input vector with independent components $\mathbf{X} \sim f_{\mathbf{X}}(\mathbf{x})=\prod_{i=1}^{M} f_{\mathbf{X}_{i}}\left(\mathbf{x}_{i}\right), \boldsymbol{\alpha}=\left(\alpha_{1}, \ldots \alpha_{M}\right)$ is a multi-index that identifies the polynomial degree in each of the input variables, and $y_{\boldsymbol{\alpha}}$ denotes the corresponding polynomial coefficient (coordinate). Following the orthonormality condition, $\mathbb{E}\left[\mathcal{M}^{\mathrm{PCE}}(\mathbf{X})\right]=y_{\mathbf{0}}$. For practical purposes, the infinite sum in (9) needs to be truncated to a finite series. This is commonly achieved by maximum-degree or hyperbolic norm truncation (for more details, see [16]). In this paper, a sparse least-square regression for the estimation of the set of coefficients $\mathbf{y}=\left\{y_{\boldsymbol{\alpha}}, \boldsymbol{\alpha} \in \mathcal{A}\right\}$ is adopted [16].

A so-called experimental design (ED), consisting of a set of realization of the input vector $\mathcal{X}=\left\{\mathbf{x}^{(1)}, \ldots, \mathbf{x}^{(N)}\right\}$ and the corresponding model evaluations $\mathcal{Y}=\left\{y^{(1)}, \ldots, y^{(N)}\right\}$, is generated first using repeated HS. In a second step, the set of coefficients $\mathbf{y}_{\alpha}$ is estimated by minimizing the expected mean-square approximation error on the ED by solving:

$$
\widehat{\mathbf{y}}_{\alpha}=\underset{\mathbf{y}_{\alpha}}{\arg \min } \mathbb{E}\left[(Y-\widehat{Y})^{2}\right]
$$

In the present application, the ED was formed by sampling the input variable space with a Sobol' lowdiscrepancy sequence. The minimization in 10 was solved using the hybrid LAR method originally proposed in [16]. A good measure of the accuracy of PCE is the mean-square residual error $\operatorname{Err}_{G}=\mathbb{E}\left[(Y-\widehat{Y})^{2}\right]$, often simply referred to as generalization error. However, the evaluation of $\operatorname{Err}_{G}$ would require an additional validation data set, which is typically unavailable. It is thus desirable to obtain an estimate of $\operatorname{Err}_{G}$ using only the information included in the ED.

One such error estimation method is the co-called leave-one-out cross-validation error (LOO). Denote by $\mathcal{M}^{P C E \backslash i}$ the PCE built from the subset of the ED obtained by removing the pair $\left\{\mathbf{x}^{(i)}, y^{(i)}\right\}$. The LOO error is defined as:

$$
\epsilon_{\mathrm{LOO}}=\frac{1}{N} \sum_{i=1}^{N}\left[\mathcal{M}\left(\mathbf{x}^{(i)}\right)-\mathcal{M}^{P C E \backslash i}\left(\mathbf{x}^{(i)}\right)\right]^{2}
$$


In the case of regression-based PCE, $\epsilon_{\mathrm{LOo}}$ can be calculated directly from the coefficients of the PCE computed with the entire ED, without explicitly solving (10) multiple times [15]. This robust error estimate allows one to devise basis-adaptive PCE schemes. A truncation scheme can be gradually relaxed, e.g. by increasing the maximum polynomial degree, until some convergence criterion on $\epsilon_{\mathrm{LOO}}$ is achieved [16]. Due to the rapid convergence of the sparse PCE coefficients, this approach provides a very efficient means to propagate the variability in the input variables to QoIs without the need for several evaluations of the model response otherwise required by standard MC techniques.

It is straightforward to obtain the Sobol' decomposition of $Y$ in an analytical form by observing that the summands $\mathcal{M}_{\mathbf{u}}^{\mathrm{PCE}}\left(\mathbf{X}_{\mathbf{u}}\right)$ in $(3)$ can be written as:

$$
\mathcal{M}_{\mathbf{u}}^{\mathrm{PCE}}\left(\mathbf{X}_{\mathbf{u}}\right)=\sum_{\alpha \in \mathcal{A}_{\mathbf{u}}} y_{\boldsymbol{\alpha}} \boldsymbol{\Psi}_{\boldsymbol{\alpha}}\left(\mathbf{X}_{\mathbf{u}}\right)
$$

where $\mathcal{A}_{\mathbf{u}}$ denotes the set of multi-indices that depend only on $\mathbf{u}$ :

$$
\mathcal{A}_{\mathbf{u}}=\left\{\boldsymbol{\alpha} \in \mathcal{A}: \alpha_{k} \neq 0 \text { if and only if } k \in \mathbf{u}\right\}
$$

Clearly, $\cup \mathcal{A}_{\mathbf{u}}=\mathcal{A}$. Consequently, due to the uniqueness of Sobol'-Hoeffding decomposition, there is an analytical expression of $\mathcal{M}_{\mathbf{u}}^{\mathrm{PCE}} 12$, which serves as a proxy of $\mathcal{M}_{\mathbf{u}}$. Due to the orthogonality of the basis, the total variance of a PCE is analytically given by [14]:

$$
D=\operatorname{Var}\left[\mathcal{M}^{\mathrm{PCE}}(\mathbf{X})\right]=\sum_{\boldsymbol{\alpha} \in \mathcal{A}} y_{\boldsymbol{\alpha}}^{2}
$$

Similarly, the partial variance $D_{\mathbf{u}}$ reads:

$$
D_{\mathbf{u}}=\operatorname{Var}\left[\mathcal{M}_{\mathbf{u}}^{\mathrm{PCE}}\left(\mathbf{X}_{\mathbf{u}}\right)\right]=\sum_{\boldsymbol{\alpha} \in \mathcal{A}_{\mathbf{u}}} y_{\boldsymbol{\alpha}}^{2} .
$$

Accordingly, the Sobol' indices of any order can be approximated by a simple combination of the squares of the PCE coefficients by substituting (15) and (14) in (8). For instance, the first-order Sobol' indices, which describe the influence of each input variable $X_{i}$ considered separately, read:

$$
S_{i}^{(1)}=\frac{\sum_{\boldsymbol{\alpha} \in \mathcal{A}_{i}} y_{\boldsymbol{\alpha}}^{2}}{\sum_{\boldsymbol{\alpha} \in \mathcal{A}} y_{\boldsymbol{\alpha}}^{2}}, \mathcal{A}_{i}=\left\{\boldsymbol{\alpha} \in \mathcal{A}: \alpha_{i}>0, \alpha_{i \neq j}=0\right\}
$$

whereas the total Sobol' indices, which represent the total effect of an input variable $X_{i}$ accounting for its main effect and all interaction with other input variables, are given by:

$$
S_{i}^{\text {(tot) }}=\frac{\sum_{\boldsymbol{\alpha} \in \mathcal{A}_{i}^{\text {tot }}} y_{\boldsymbol{\alpha}}^{2}}{\sum_{\boldsymbol{\alpha} \in \mathcal{A}} y_{\boldsymbol{\alpha}}^{2}}, \mathcal{A}_{i}^{\mathrm{tot}}=\left\{\boldsymbol{\alpha} \in \mathcal{A}: \alpha_{i}>0\right\} .
$$

Sobol' indices provide quantitative insight on the importance of an input variable. However, they do not include information about the direction in which an input variable affects the QoI. So-called univariate effects can answer this question [24, 18. A univariate effect is the expectation of a QoI conditioned to the value of a single input variable. Univariate effects have a closed analytical form for PCE models, which is closely related to the first-order Sobol' decomposition:

$$
\mathcal{M}_{i}^{(1)}\left(X_{i}\right)=\sum_{\boldsymbol{\alpha} \in \mathcal{A}_{i}} y_{\boldsymbol{\alpha}} \Psi_{\boldsymbol{\alpha}}\left(X_{i}\right), \mathcal{A}_{i}=\left\{\boldsymbol{\alpha} \in \mathcal{A}: \alpha_{i}>0, \alpha_{i \neq j}=0\right\}
$$

In this paper, computations of both PCE and Sobol' indices are performed using UQLAB, which is a MATLAB toolbox for uncertainty quantification developed by the Chair of Risk, Safety and Uncertainty Quantification of ETH Zurich [25, 26, 27].

\section{Case study}

In order to provide experimental validation of the proposed GSA framework for HS, a case study was conducted using a 3-DoFs prototype structure subjected to earthquake ground motion excitation. 


\subsection{Prototype structure and excitation}

The prototype structure consists of a simply-supported massive beam coupled at the ends to two linear-elastic rotation restraints, two lumped masses, and one dashpot. The beam is subjected to a ground motion excitation $a_{g}(t)$ acting in the direction orthogonal to its axis. Bending and axial deformation of the beam are coupled for large displacements, which means that the prototype structure is characterized by a geometrical nonlinearity. Figure 1 provides a schematic view of the prototype structure and its hybrid model substructured into one PS and two NS for the purpose of HS.

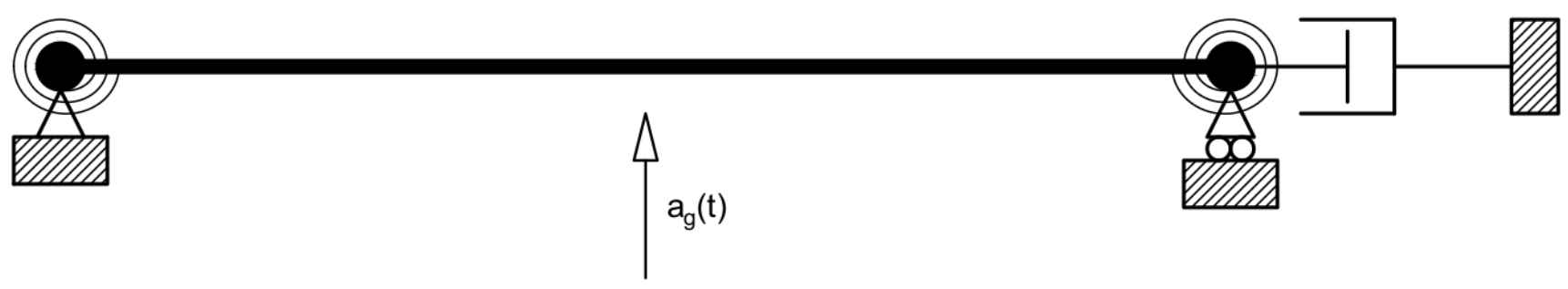

(a)
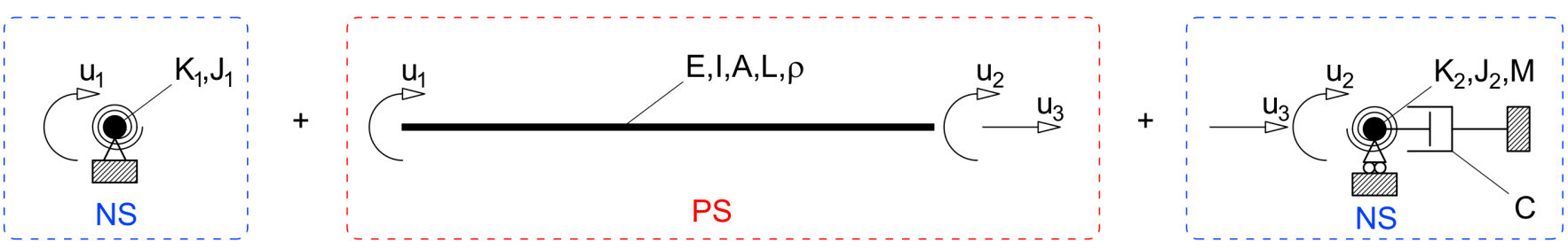

(b)

Figure 1: Case study: (a) prototype structure; (b) hybrid model comprising two NS and one PS.

In Figure $1 u_{1}$ and $u_{2}$ indicate the two rotational DoFs of the simply-supported beam, while $u_{3}$ corresponds to its axial DoF. The PS is instantiated using a $L=470 \mathrm{~mm}$ long iron plate (Young modulus $E=100 \mathrm{GPa}$, density $\rho=7850 \frac{\mathrm{kg}}{\mathrm{m}^{3}}$ ), with a $200 \times 2 \mathrm{~mm}$ cross-section (area $A=400 \mathrm{~mm}^{2}$ and moment of inertia $I=133 \mathrm{~mm}^{4}$ ). Since HSs are conducted with a testing time scale equal to 50, a numerical mass matrix accounts for the PS inertia. In detail, the PS mass matrix is estimated by condensing the six-by-six consistent mass matrix of a two-dimensional Bernoulli beam element to the three DoFs retained by the hybrid model. The out-of-diagonal entries of the reduced mass matrix couple the inertial acceleration of the ground motion to the rotation DoFs of the hybrid model. The NS of the hybrid model comprises two rotational masses $J_{1}=J_{2}=0.9 \mathrm{kgm}^{2}$, a translation mass $M=20,000 \mathrm{~kg}$ all lumped at the supports. The linear dash-pot with a damping coefficient $C=300,000 \frac{\mathrm{Ns}}{\mathrm{m}}$ is the only source of numerical damping and prevents the excitation of the axial eigenmode of the hybrid model. The two linear elastic rotation springs, whose stiffness $K_{1}$ and $K_{2}$ are defined as input variables for GSA, represent the non-deterministic properties of the prototype structure supports.

The velocity-pulse model proposed by [28], which provides an intuitive representation of near-fault earthquake ground motions, defines the excitation $a_{g}(t)$. The velocity-pulse model consists of a cosine carrier wave with a period $T_{p}$, modulated by a truncated cosine function, namely the pulse function, with a period $\gamma T_{p}$ and amplitude $V_{p}$. The latter corresponds to the peak velocity of the pulse occurring at time $t_{\max , p}$. The expression for the velocity-pulse reads:

$$
v_{g}(t)=\left(\frac{V_{p}}{2} \cos \left(2 \pi\left(\frac{t-t_{\max , p}}{T_{p}}\right)+\nu\right)-\frac{D_{r}}{\gamma T_{p}}\right)\left(1+\cos \left(\frac{2 \pi}{\gamma}\left(\frac{t-t_{\max , p}}{T_{p}}\right)\right)\right),
$$

where $\nu$ is the phase angle between the cosine wave and pulse function, while $D_{r}$ is the non-zero residual displacement at the end of the pulse, which is in turn given by:

$$
D_{r}=V_{p} T_{p} \frac{\sin (\nu+\gamma \pi)-\sin (\nu-\gamma \pi)}{4 \pi\left(1-\gamma^{2}\right)} .
$$

The transverse inertial acceleration $a_{g}(t)$ applied to the prototype structure corresponds to the time derivative of the velocity-pulse model. The velocity-pulse model is defined for $t \in\left\{0, \gamma T_{p}\right\}$ and is zero outside this interval. 


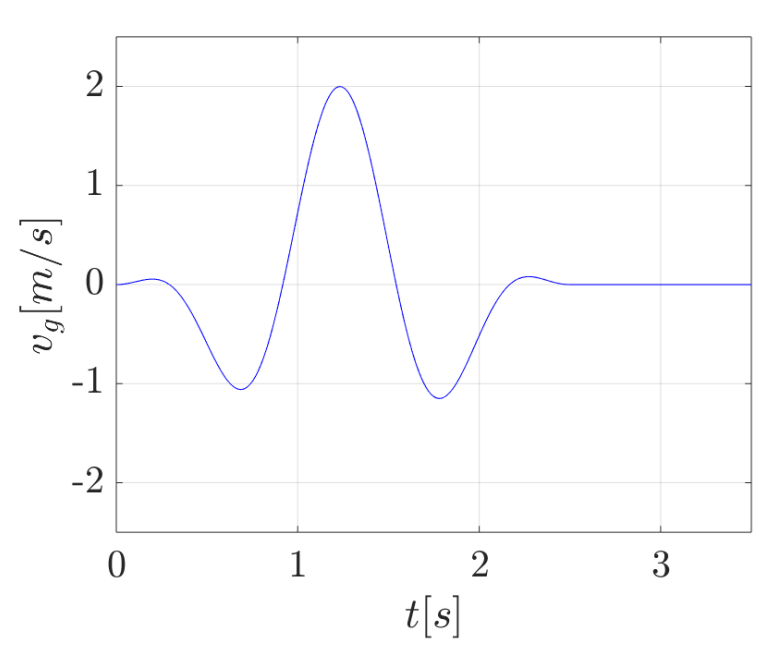

(a)

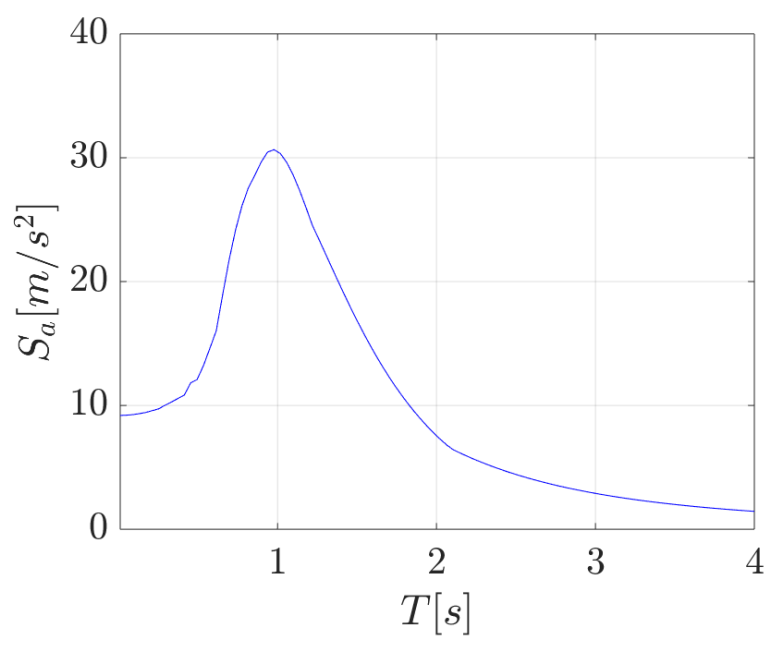

(b)

Figure 2: Sample excitation used in the case study: (a) Velocity-pulse time history, and (b) the corresponding acceleration response spectrum $S_{a}$ computed using a $5 \%$ damping ratio.

Table 1: Input variable intervals and assumed probability distributions.

\begin{tabular}{|c|c|c|c|c|}
\hline Parameter & Probability distribution & Lower bound & Upper bound & Unit \\
\hline$K_{1}$ & Uniform & 20 & 120 & {$\left[\frac{\mathrm{Nm}}{\mathrm{rad}}\right]$} \\
\hline$K_{2}$ & Uniform & 20 & 120 & {$\left[\frac{\mathrm{Nm}}{\mathrm{rad}}\right]$} \\
\hline$V_{p}$ & Uniform & 3.50 & 4.50 & {$\left[\frac{\mathrm{m}}{\mathrm{s}}\right]$} \\
\hline$T_{p}$ & Uniform & 0.50 & 1.50 & {$[\mathrm{~s}]$} \\
\hline \multicolumn{4}{|r}{} \\
\end{tabular}

In order to reduce the number of free parameters, the velocity pulse peak is located at $t_{\text {max }, p}=\frac{\gamma T_{p}}{2}$ and $\gamma=2$ and $\nu=0.1$. Thus, pulse peak velocity $V_{P}$ and period $T_{P}$ are selected as input variables for GSA. Figure 2 depicts a realization of the velocity-pulse model described by (19) and (20) with $V_{P}=2 \frac{\mathrm{m}}{\mathrm{s}}, T_{P}=1.25 \mathrm{~s}, \gamma=2.0$ and $\nu=0.1 \mathrm{rad}$.

The GSA of the hybrid model is evaluated using two response QoIs, $u_{1, \max }$, the maximum absolute rotation at the left support of the beam measured using the vertical actuator displacement, and $r_{1, \max }$, the maximum absolute bending moment at the left support of the beam measured using the vertical actuator load cells, as depicted in Figure 1. Following the notation introduced in Section 2, the vector of input variables and the vector of QoIs read:

$$
\begin{aligned}
& \mathbf{X}=\left\{V_{P}, T_{P}, K_{1}, K_{2}\right\} \\
& \mathbf{Y}=\left\{u_{1, \max }, r_{1, \max }\right\}
\end{aligned}
$$

The input variables uniformly distributed. In particular, the intervals reported in Table 1 entail a linear response of the hybrid model, which is compatible with the force and displacement capacity of the HS test setup.

If $K_{1}=K_{2}$ and both vary within the range proposed in Table 1, the period of the first bending mode of the hybrid model ranges between 0.68 and $0.45 \mathrm{~s}$. This range overlaps with the period range of the carrier signal of the velocity-pulse excitation, which varies between 0.50 and $2.00 \mathrm{~s}$. Accordingly, the variable frequency tuning between the excitation and the first bending eigenmode of the hybrid model is expected to affect the dynamic amplification of both response QoIs.

There are two reasons for setting the domains of input variables to ensure a linear elastic PS behavior. Firstly, this choice allows for performing the entire experimental campaign using a single plate specimen as PS. Second, the case study focuses on the measurement noise as the sole source of uncertainty that affects the intra-experiment variability of the PS response. Ultimately, the proposed GSA method for HS could be validated in this case study. 


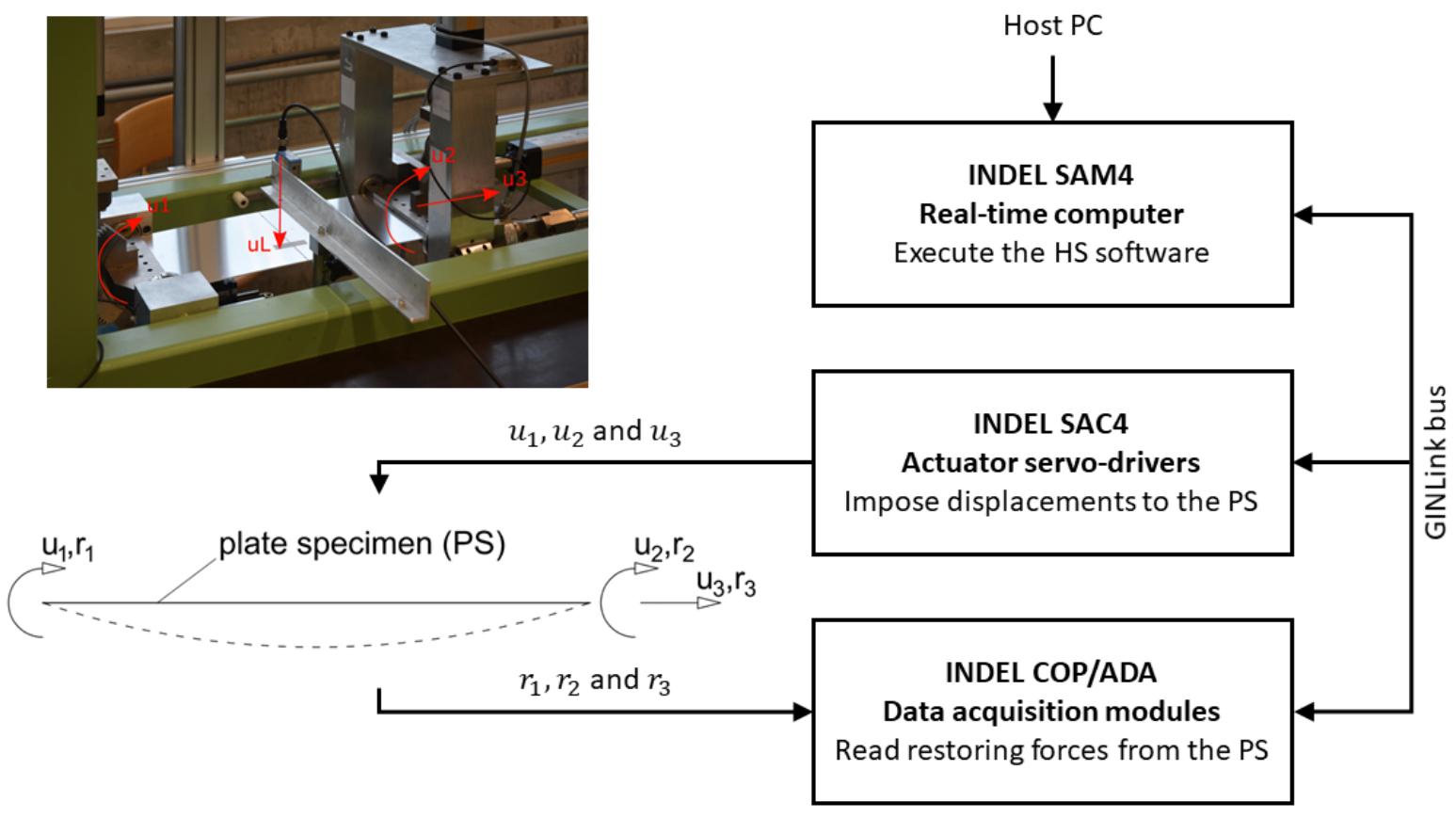

Figure 3: Architecture of the 3-DoFs HS test rig.

\subsection{Hybrid simulation setup}

The 3-DoFs HS test rig used to conduct the HSs is a stiff loading frame equipped with four electro-mechanical actuators interfaced to an INDEL real-time computer [29]. The 3-DoFs HS test rig is designed to test plate specimens with an approximate footprint of $200 \times 500 \mathrm{~mm}$ and thickness varying between 1 and $3 \mathrm{~mm}$. Figure 3 illustrates the architecture of the HS setup, including a close-up view of the plate specimen accommodation. The GINLink bus connects the actuator servo-driver INDEL SAC4 and all data acquisition modules INDEL COP-ADA to the real-time computer INDEL SAM4, which runs the HS software. The latter is developed in the MATLAB/SIMULINK environment and downloaded to the real-time computer INDEL SAM4 via Ethernet from the Host-PC. At each simulation time step, the HS software imposes displacements $u_{1}, u_{2}$ and $u_{3}$ to the plate specimen, the PS, reads the corresponding restoring forces $r_{1}, r_{2}$ and $r_{3}$ measured using force transducers, and solves the coupled equation of motion of the hybrid model. In addition, a laser sensor measures the outof-plane deflection at the mid-span of the plate specimen (labeled UL in Figure 3). A detailed description of the time integration scheme adopted to solve the equation of motion is reported in the appendix of this paper. Figure 4 shows two axonometric views of the 3-DoFs HS test rig, including the main hardware components. In this figure, the gray-colored parts are fixed to the reaction frame, which is not visible, while the moving parts of the 3-DoF HS test rig are colored in yellow. The two rack-pinion systems (10) convert motion along the vertical actuator axes $y_{1}$ and $y_{2}(1)$ to rotations $u_{1}$ and $u_{2}$, respectively, which are imposed to the short edges of the plate specimen (6) through aluminum clamps (3). Horizontal actuators along axes $x_{1}$ and $x_{2}(2)$ control the position of the moving frame (4), which is mounted on two profiled rail guides using ball bearings (5). As a result, the position of the moving frame (4) equals the axial elongation of the plate specimen $u_{3}(6)$.

\section{Case study results}

This section reports the results of the case study where the GSA procedure described in Section 2 was employed to evaluate and quantify the sensitivities of the response of the prototype structure defined in Section 3 to earthquake ground motion excitation using the results of a number of HS conducted using the 3-DoFs test rig described in Section 3 . 

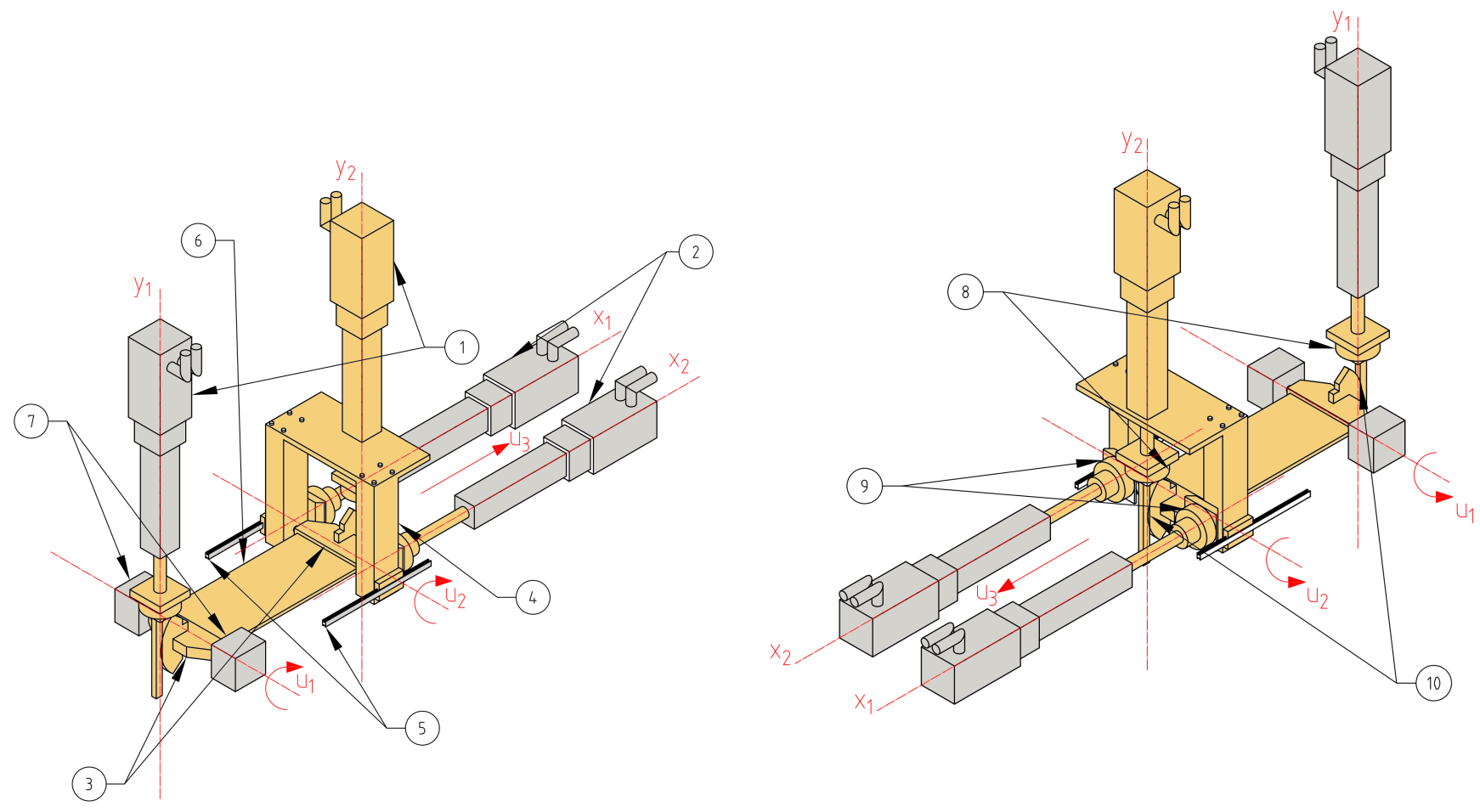

Figure 4: Axonometric views of the 3-DoFs HS test rig with main components: (1) vertical actuators; (2) horizontal actuators; (3) installation clamps; (4) moving frame; (5) profiled rail guides; (6) plate specimen; (7) hinges; (8) vertical actuator load cells; (9) horizontal actuator load cells; (10) rack-pinion systems. The moving parts are colored in yellow while the grey parts are fixed to the reaction frame, which is omitted in this figure for clarity.

\subsection{Surrogate modeling using PCE}

Based on the input variable intervals and distributions specified in Table 1, a Sobol' sequence was used to generate 200 samples of the input variable vector $\mathbf{X}$. For each of these samples, a HS of the response of the hybrid model (Figure 1) of the prototype structure to a velocity-pulse ground motion excitation was conducted to determine the time history of the response QoIs in vector $\mathbf{Y}$. An outcome of a sample HS of the response of the prototype structure with support rotation spring stiffness $K_{1}=K_{2}=70 \mathrm{Nm} / \mathrm{rad}$ to the velocity pulse shown in Figure 2 in terms of time histories of the rotation $u_{1}(t)$ and moment $r_{1}(t)$ is reported in Figure 5. where the corresponding peak response QoIs $u_{1, \max }$ and $r_{1, \max }$ are also highlighted. According to the procedure outlined in Section 2.2. ED was used to train the PCEs of response QoI $u_{1, \max }$ and $r_{1, \max }$.

The time history of support moment $r_{1}$ is much noisier than that of support rotation $u_{1}$. In principle, when the stiffness of the specimen is much smaller than the stiffness of the actuation system, displacement control is fairly accurate, and restoring force measurement noise is the dominant component of experimental errors, which directly propagates to the acceleration response history of the hybrid model. Double integration from acceleration to displacement, done by the dynamic equation of motion of the hybrid model, acts as a low-pass filter that reduces the impact of noise on displacement measurements. As a consequence, measurement signal noise affects $r_{1, \max }$ more than it does $u_{1, \max }$ and, further negatively affects the convergence of the corresponding PCE. Validation scatter plots of Figure 6 compare the measured QoIs to the corresponding PCE predictions and confirm this hypothesis. In detail, for each QoIs, four PCEs were trained on four disjoint 150-sample partitions of the ED and evaluated on the remaining 50 samples.

Figure 7 shows the convergence of LOO errors of both PCEs computed considering EDs of increasing size from 10 to 200 samples. It is worth noting that force measurement noise and displacement control errors are both sources of aleatory uncertainty that propagate through the hybrid model response, which is inherently stochastic. In this case, PCE acts as a data denoiser, and for this reason, LOO errors do not converge to zero. Figures 8 and 9 show the convergence plots of the mean, variance and CoV PCE-based estimates for both QoIs. Evidently, all PCE estimates converge in a stable manner, with reliable values attained for ED sizes larger than 50 samples. 


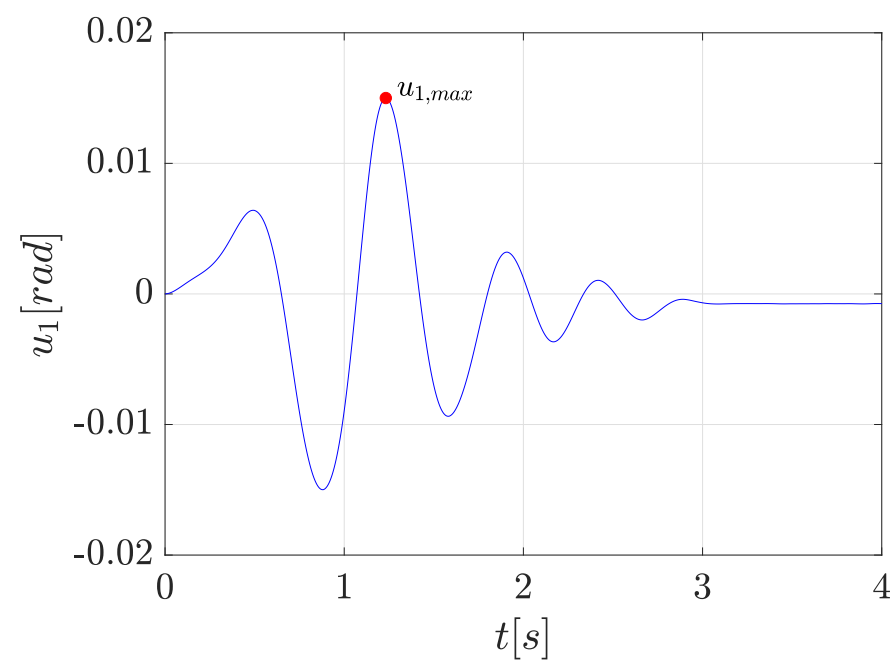

(a)

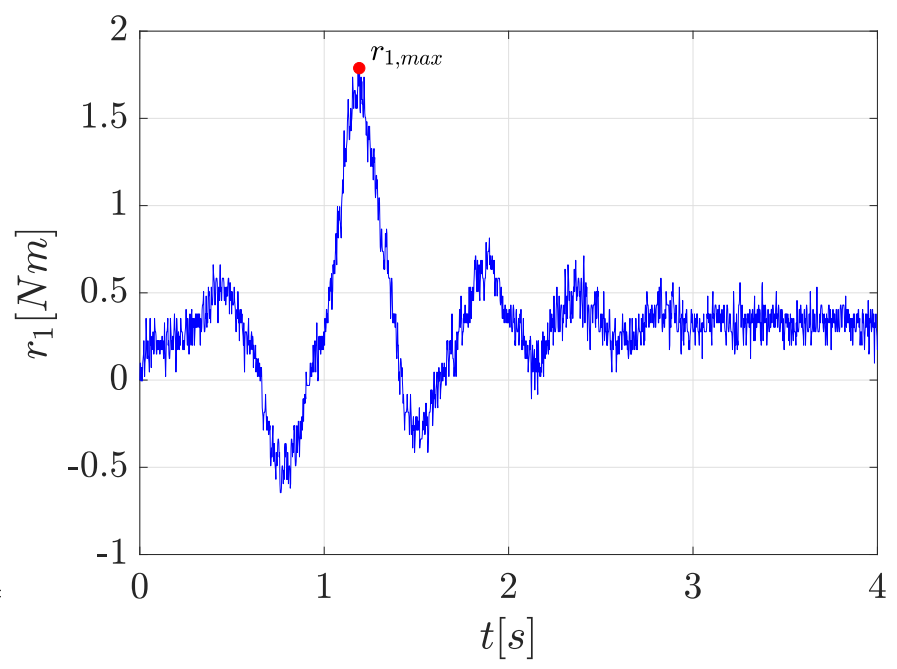

(b)

Figure 5: Outcomes of a sample HS of the response of the hybrid model of the prototype structure with $K_{1}=K_{2}=70 \mathrm{Nm} / \mathrm{rad}$ to a velocity-pulse ground motion excitation with $V_{P}=2 \mathrm{~m} / \mathrm{s}, T_{P}=1.25 \mathrm{~s}$ in terms of left support rotation (a) and bending moment (b) response histories. QoIs are highlighted.

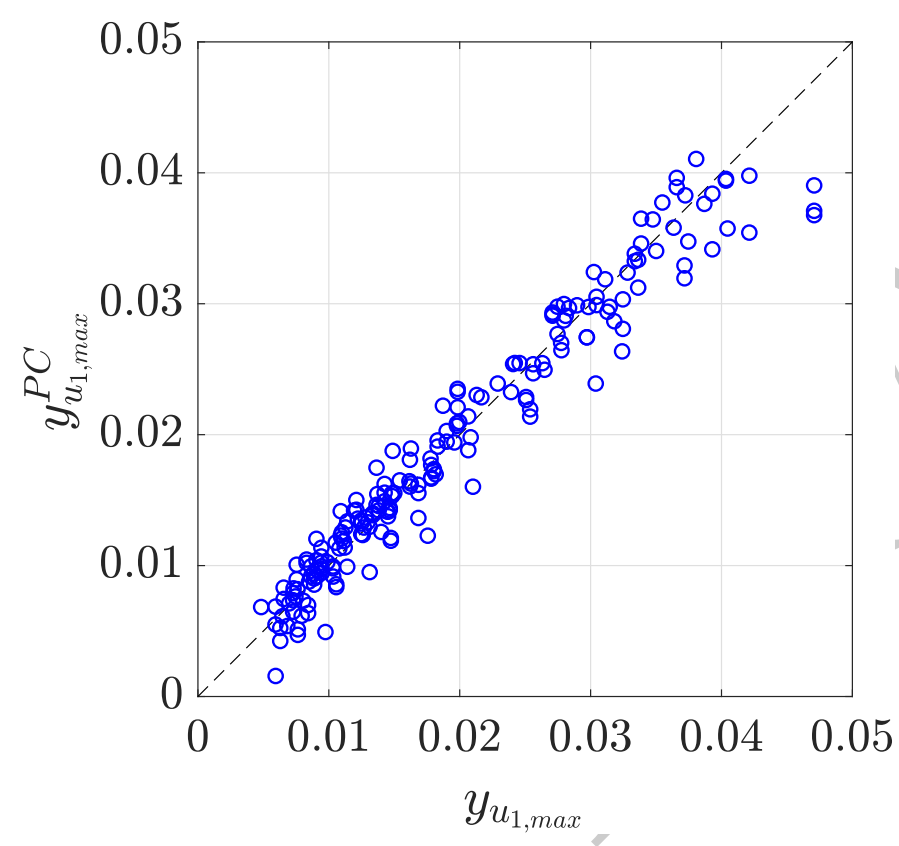

(a)

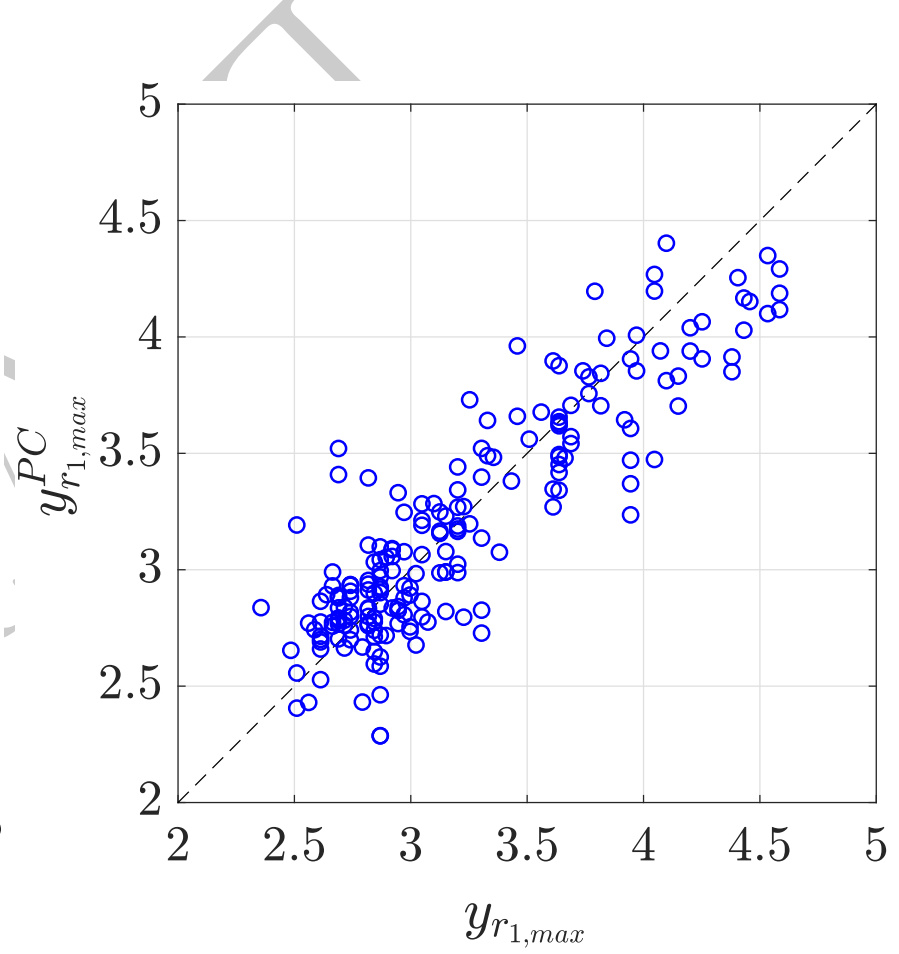

(b)

Figure 6: Comparison between QoI measurements and corresponding PCE estimates for (a) $u_{1, \max }$ and (b) $r_{1, \max }$.

\subsection{GSA based on Sobol' sensitivity indices}

Based on PCEs of both QoIs, Sobol' indices are obtained as explained in Subsection 2.2. Figure 10 reports firstorder and total Sobol' indices for QoIs $u_{1, \max }$ and $r_{1, \max }$. The error bars of Figure 10 refer to $95 \%$ confidence intervals and are computed from 50 bootstrap replications of the underlying PCE by using the percentile method introduced in [30]. It is important to remark that there is no theoretical guarantee that the percentile-based method provides unbiased quantile estimates. However, as discussed and demonstrated in [30], bias decays quickly with PCE, and, for a large enough ED (30-50 points), bootstrap provides an effective estimator of the accuracy of the Sobol' indices.

According to Figure 10, the period $T_{p}$ of the velocity pulse and the stiffness $K_{1}$ of the left torsional spring are the most sensitive input variables for both QoIs. This result finds an intuitive explanation. The range of velocity-pulse period $T_{p}$ was selected to cross the range of periods of the first bending mode of the hybrid 


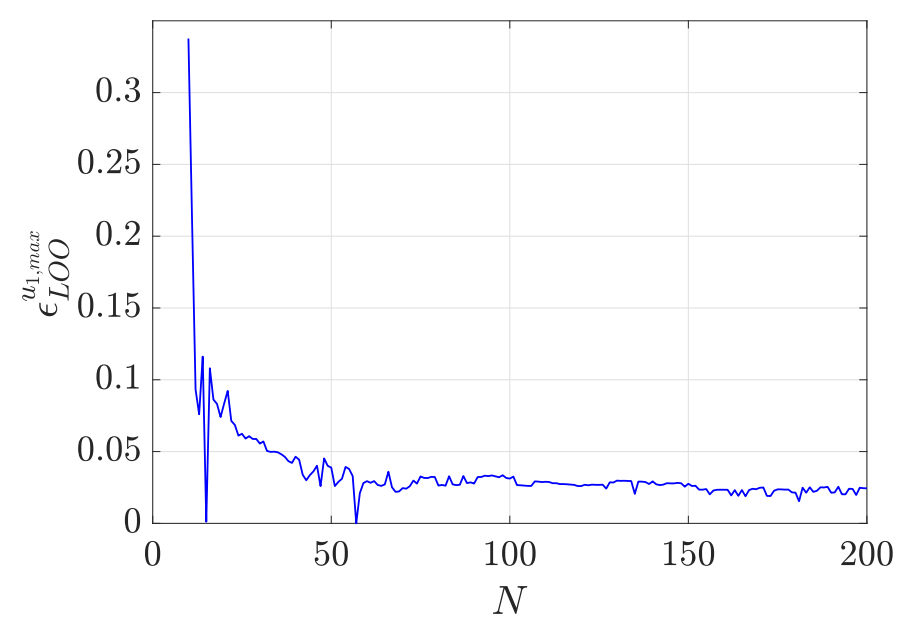

(a)

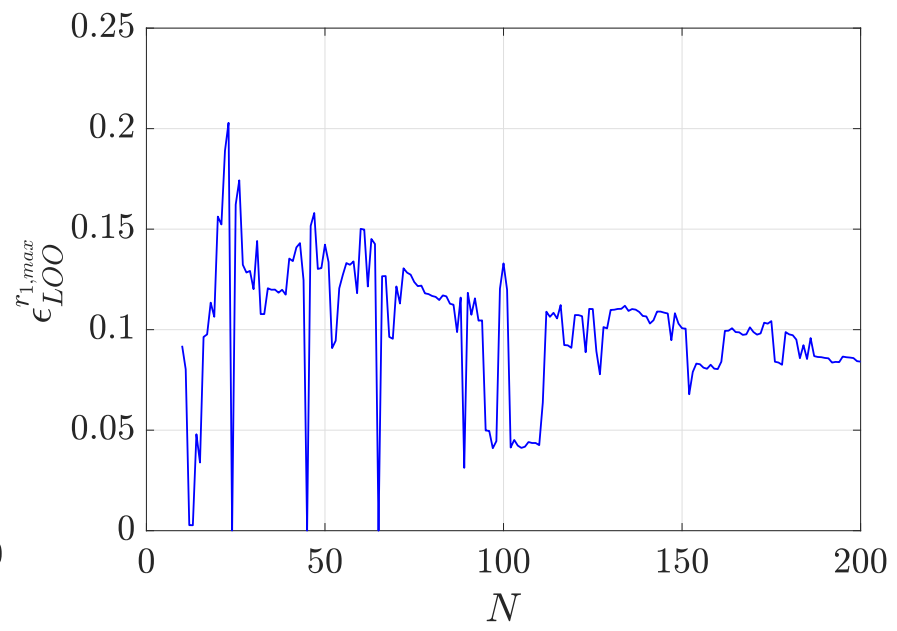

(b)

Figure 7: Convergence of LOO error estimates of (a) $u_{1, \max }$ and (b) $r_{1, \max }$ PCEs.

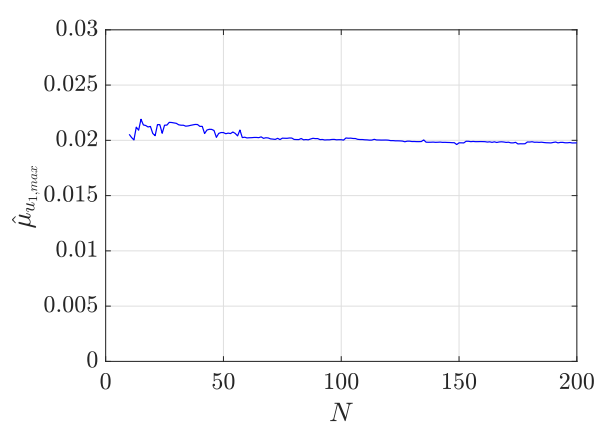

(a)

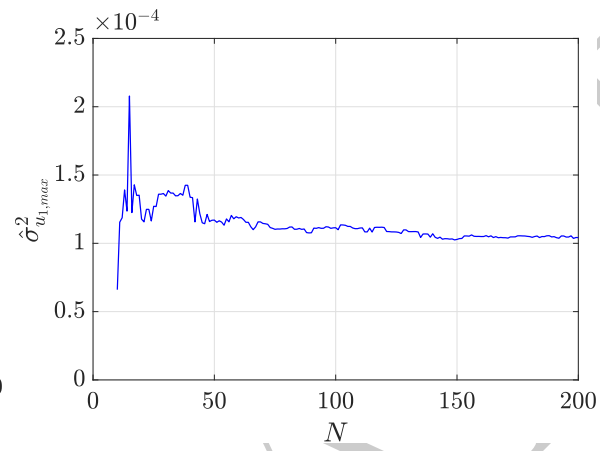

(b)

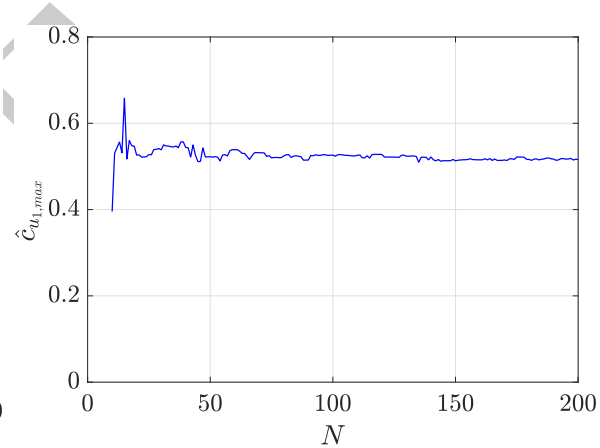

(c)

Figure 8: Convergence of PCE estimates of (a) mean, (b) variance and (c) CoV of QoI $u_{1, \max }$.

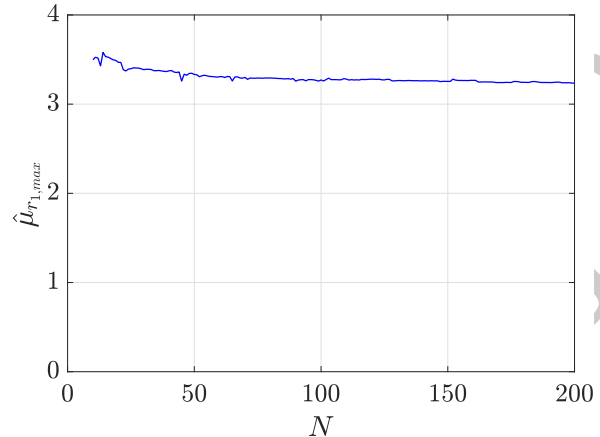

(a)

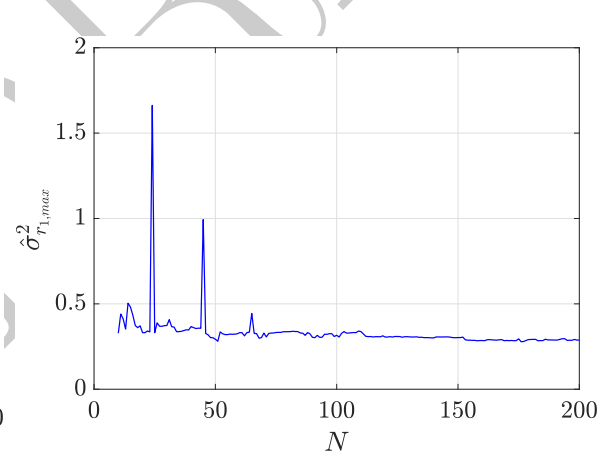

(b)

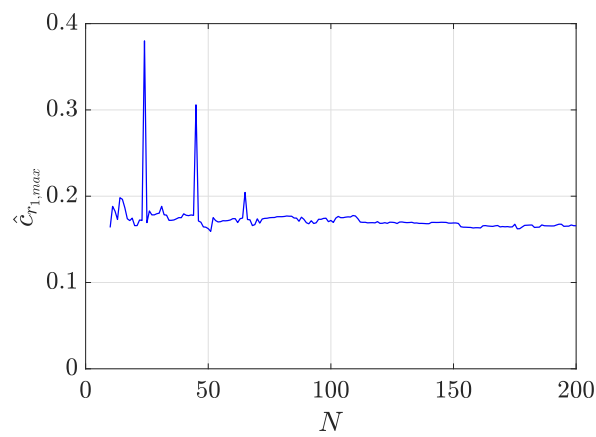

(c)

Figure 9: Convergence of PCE estimates of (a) mean, (b) variance and (c) CoV of QOI $r_{1, \max }$.

model, given the range of the stiffness of the rotation springs. Further, rotation spring stiffness $K_{1}$ is a boundary condition at the left edge of the PS iron plate, corresponding to responses $u_{1}$ and $r_{1}$. Therefore, QoIs are expected to be more sensitive to $K_{1}$ than $K_{2}$. It should be noted that the bending stiffness of the plate specimen has the same order of magnitude as the maximum stiffness value of the left-support rotation spring $K_{1}$ (see Table 1), thus a fixed boundary configuration, which would heavily affect the response of the plate, was not approached in this case study. It is, indeed, reasonable from the standpoint of resonance that the velocity-pulse period $T_{P}$ has more influence on the prototype structure response than $K_{1}$, and significantly more influence than the velocity pulse amplitude $V_{P}$. Finally, the small difference between pairs of first-order and total Sobol' indices indicates that the high-order interactions among input variables are negligible.

In order to provide further insight into the prototype structure response, univariate effects are reported in 


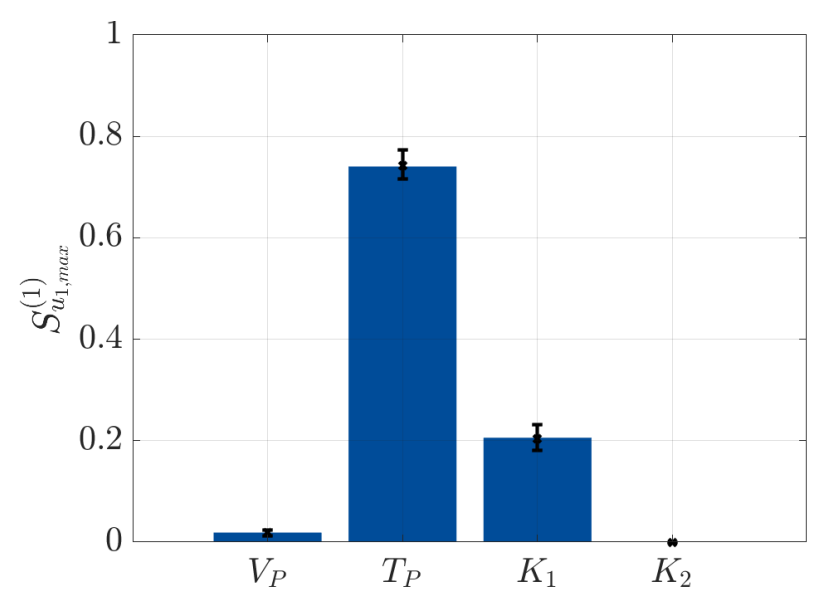

(a)

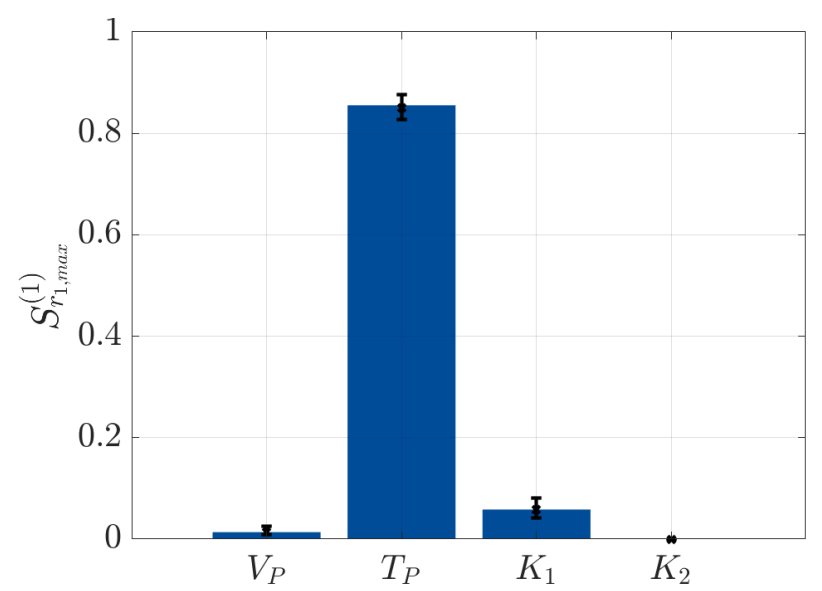

(c)

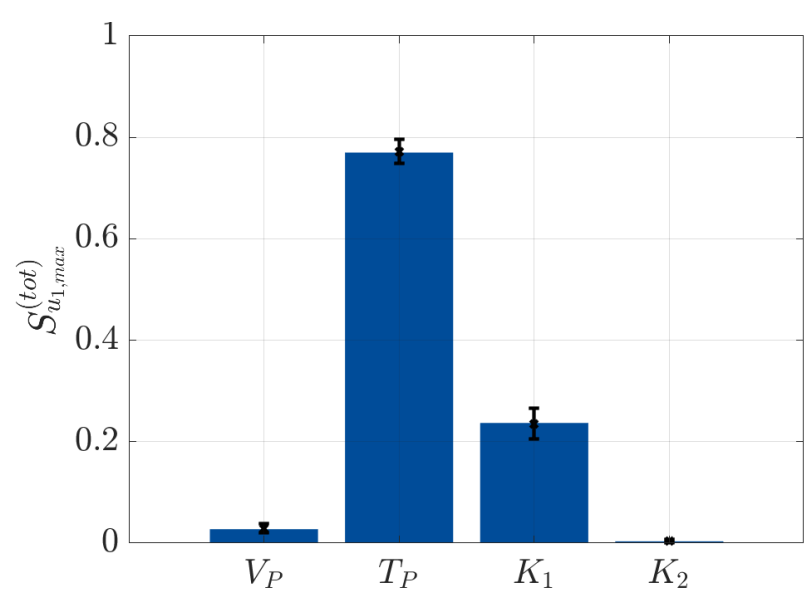

(b)

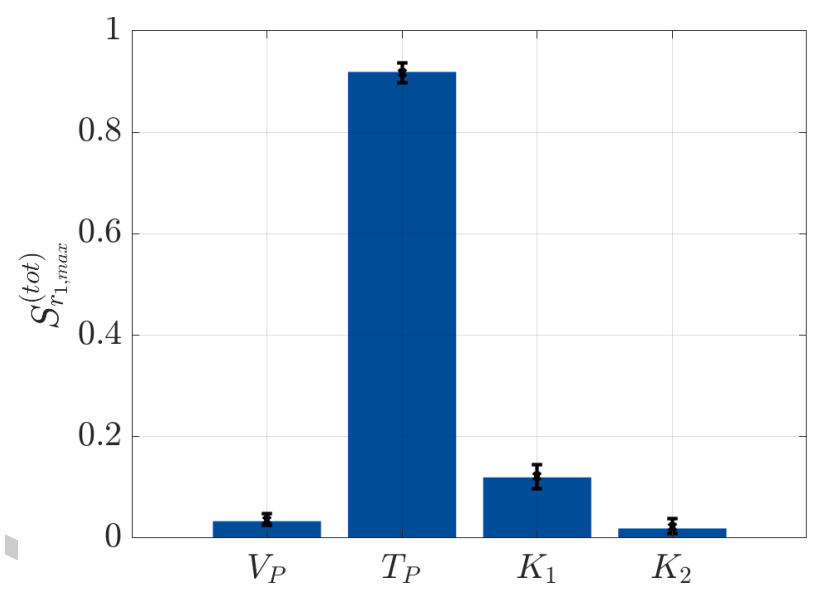

(d)

Figure 10: First-order and total Sobol' indices for (a-b) $u_{1, \max }$ and (c-d) $r_{1, \max }$ PCEs.

Figure 11 for input variables $T_{P}$ and $K_{1}$, to which the response of the prototype structure is the most sensitive, as highlighted by the GSA. Related $95 \%$ confidence intervals computed with 50 bootstrap replications of the underlying PCEs are also reported in the same figures. Evidently, univariate effects decrease monotonically for PCEs of both QoIs over the ranges of the two considered input variables.

\section{Conclusions}

A Global Sensitivity Analysis (GSA) framework for Hybrid Simulation (HS) was presented in this paper. The proposed framework, based on Sobol' sensitivity indices, is intended to quantify the fraction of the variability of a generic response quantity of interest (QoI) of the prototype structure due to the variability of a set of input variables that provide a suitable parametrization of the hybrid model and the excitation. The response of the prototype structure is evaluated using HS, thus involving a hybrid model comprised of a few Physical Substructures (PSs) tested in laboratory test setups, and a potentially large number of Numerical Substructures (NSs), emulated using computer models. Such an approach provides for a more realistic evaluation of the response of the prototype structure but still precludes the conduct of a large number of tests due to cost and time considerations. To resolve this issue, Polynomial Chaos Expansion (PCE) was used to surrogate the hybrid model response. Thereafter, Sobol' sensitivity indices were obtained as a by-product of polynomial coefficients, entailing a reduced number of HSs compared to a crude Monte Carlo approach to achieve the same quality of response sensitivity estimates. A case study characterized by four uncertain input variables, two of which related to the NS while the other two related to the excitation, was used to validate the proposed framework.

As a general observation, the noise floor of each response QoI of the prototype structure, which is determined by random experimental errors entailed in HS, prevents the generalization error of the corresponding PCE to converge to zero asymptotically. In the case of a soft specimen, such as the one used in the case study, 


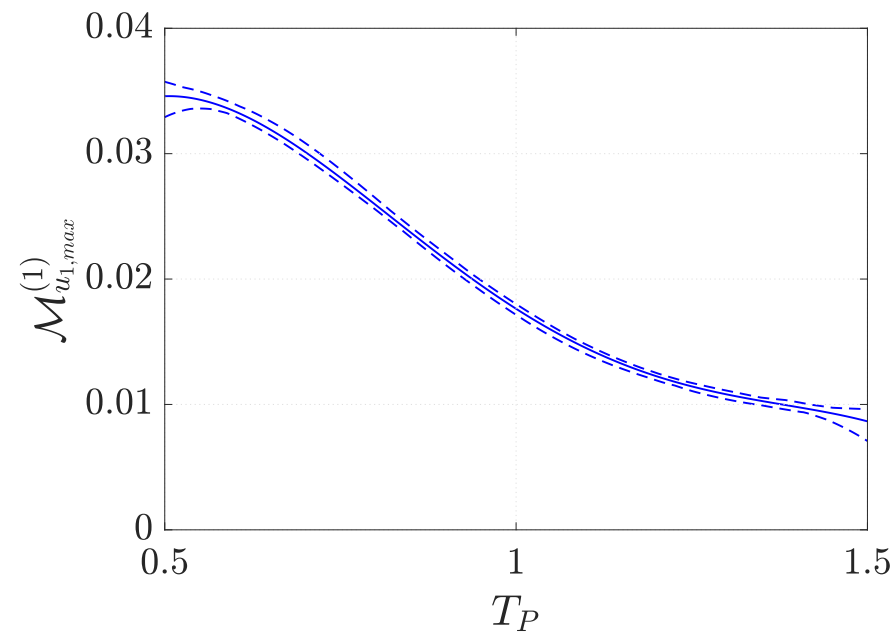

(a)

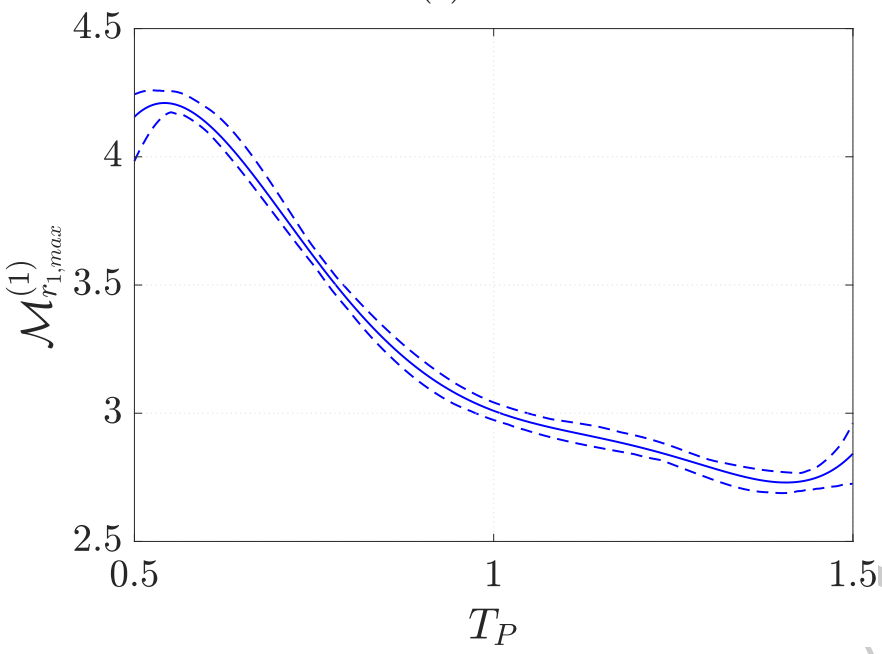

(c)

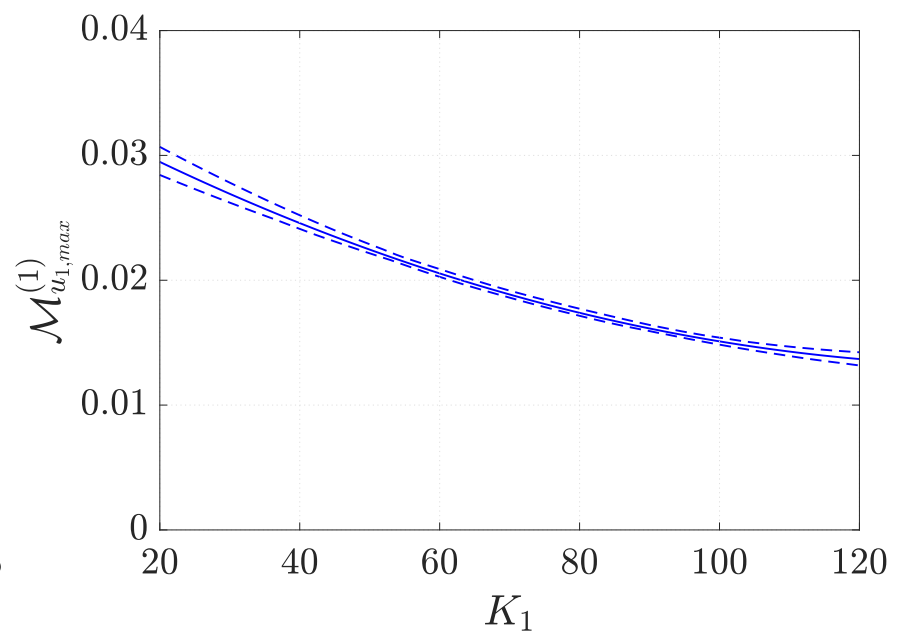

(b)

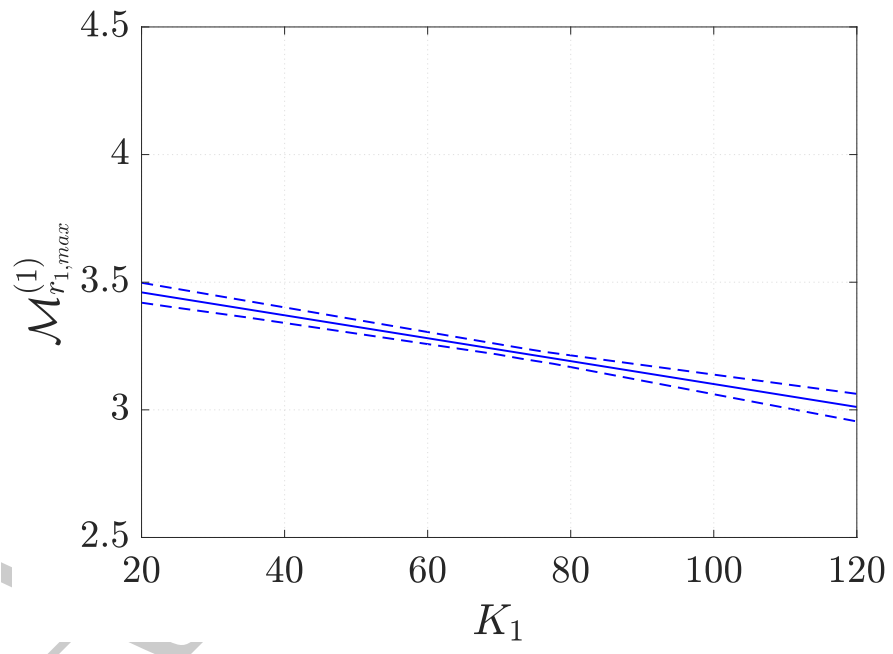

(d)

Figure 11: Univariate effects on PCEs for (a-b) $u_{1, \max }$ and (c-d) $r_{1, \max }$. Dashed lines indicate $95 \%$ confidence intervals.

displacement-based QoIs are less noisy than force-based QoIs. However, stable plateau values indicate that PCE acts as a powerful denoiser and, therefore, is very well-suited to surrogate the hybrid model response, making the proposed GSA framework for HS possible. Stable PCEs and, hence, stable Sobol' sensitivity indices, were available with as few as $50 \mathrm{HSs}$ in the conducted case study. Several such HSs can be easily afforded when the PSs of the hybrid model can sustain repeated experiments without deteriorating appreciably (e.g. seismic isolation bearings). However, when the PSs accumulate damage during HS and need to be replaced experiment after experiment, several such HSs might not be affordable. Therefore, future work should aim to reduce the number of HSs necessary to achieve stable convergence of PCEs by, for example, employing adaptive sampling strategies to build the ED.

GSA adds a further dimension to HS to make it into a tool for exploring the dynamic response of the prototype structure in the domain of engineering relevance of the prototype structure response. Namely, a functional understanding of the hybrid model response to a dynamic excitation is sought (e.g. what influences what and how much?) within a domain defined by reasonable variation of the input parameters. Furthermore, GSA enables quantification of the impact of experimental uncertainties on surrogate models, a technique not documented in the literature to the best knowledge of the authors, opening a new field of stochastic HS in structural earthquake engineering.

Finally, the findings of the presented application example indicate that experimental campaigns designed according to the current earthquake engineering practice and, therefore, relying on a few HSs with degrading PSs, can only provide qualitative instead of quantitative information about the structural sensitivity of the prototype structure response. Yet, quantifying the response sensitivity is very valuable, both in terms of planning additional HSs for model validation and verification, and in terms of modeling and analysis choices in the overall structural design process. This motivates our continuing work. 


\section{Appendix A. Time integration algorithm for hybrid simulation}

A partitioned time integration algorithm tailored to state-space equations of motion computes the time history response of the hybrid model in a HS. The state-space formulation of the equation of motion of a generic mechanical system reads:

$$
\mathbf{M} \dot{\mathbf{Y}}+\mathbf{R}(\mathbf{Y})=\mathbf{F}(t)
$$

with,

$$
\mathbf{Y}=\left[\begin{array}{c}
\mathbf{u} \\
\mathbf{v} \\
\mathbf{s}
\end{array}\right], \mathbf{M}=\left[\begin{array}{ccc}
\mathbf{I} & \mathbf{0} & \mathbf{0} \\
\mathbf{0} & \mathbf{m} & \mathbf{0} \\
\mathbf{0} & \mathbf{0} & \mathbf{I}
\end{array}\right], \mathbf{R}=\left[\begin{array}{c}
-\mathbf{v} \\
\mathbf{r}(\mathbf{u}, \mathbf{v}, \mathbf{s}) \\
\mathbf{g}(\mathbf{u}, \mathbf{v}, \mathbf{s})
\end{array}\right], \mathbf{F}(t)=\left[\begin{array}{c}
\mathbf{0} \\
\mathbf{f}(t) \\
\mathbf{0}
\end{array}\right]
$$

where vectors $\mathbf{u}, \mathbf{v}$ and $\mathbf{s}$ contain displacement, velocity and additional state variables, respectively. The former two vectors always appear as a pair in second-order mechanical systems, while the latter is used to model nonlinearities endowed with memory, e.g. hysteresis. In particular, $\mathbf{r}(\mathbf{u}, \mathbf{v}, \mathbf{s})$ is the nonlinear restoring force vector while $\mathbf{g}(\mathbf{u}, \mathbf{v}, \mathbf{s})$ is a nonlinear function that describes the evolution of additional state variables $\mathbf{s} ; \mathbf{m}$ is the mass matrix and $\mathbf{f}(t)$ is the external time varying load, while $\mathbf{I}$ and $\mathbf{0}$ are identity and zero matrices, respectively. When A.1 - A.2 refer to a linear system, no additional state variable s enters the state vector $\mathbf{Y}$ and the restoring force is defined as:

$$
\mathbf{r}(\mathbf{u}, \mathbf{v})=\mathbf{k u}+\mathbf{c v}
$$

where $\mathbf{k}$ and $\mathbf{c}$ are stiffness and damping matrices, respectively. For the sake of simplicity, time dependency is omitted hereafter while $\mathbf{M}, \mathbf{K}, \mathbf{R}$ and $\mathbf{F}$ are referred to as generalized mass, stiffness, restoring force and external loading.

The partitioned time integration algorithm, which adopts the dual assembly strategy of the modified Prakash-Hjelmstad method [31], synchronizes two monolithic time integration processes characterized by different time steps. In detail, a set of Lagrange multipliers enforces compatibility of PS and NS interface velocities at the coarser time step. The corresponding coupled system of equations of motion reads:

$$
\left\{\begin{array}{l}
\mathbf{M}^{N} \dot{\mathbf{Y}}_{k+1}^{N}+\mathbf{R}^{N}\left(\mathbf{Y}_{k+1}^{N}\right)=\mathbf{F}_{k+1}^{N}+\mathbf{B}^{N^{T}} \boldsymbol{\Lambda}_{k+1} \\
\mathbf{M}^{P} \dot{\mathbf{Y}}_{k+\frac{j}{m}}^{P}+\mathbf{R}^{P}\left(\mathbf{Y}_{k+\frac{j}{m}}^{P}\right)=\mathbf{F}_{k+\frac{j}{m}}^{P}+\mathbf{B}^{P^{T}}\left(\frac{m-j}{m} \boldsymbol{\Lambda}_{k}+\frac{j}{m} \boldsymbol{\Lambda}_{k+1}\right) \\
\mathbf{G}^{N} \dot{\mathbf{Y}}_{k+1}^{N}+\mathbf{G}^{P} \dot{\mathbf{Y}}_{k+1}^{P}=\mathbf{0}
\end{array}\right.
$$

where signed Boolean collocation matrices $\mathbf{B}$ and $\mathbf{G}$ localize interface forces and define compatibility equations, respectively, and are defined as:

$$
\mathbf{B}=\left[\begin{array}{lll}
\mathbf{0} & 1 & 0
\end{array}\right], \mathbf{G}=\left[\begin{array}{lll}
1 & 0 & 0
\end{array}\right]
$$

Boolean collocation matrices 1 localize interface DoF pairs across NS and PS. In principle, at each simulation step, the computation of the state vector A.4 is split into free and link solutions. The coupled solution is the sum of free and link contributions. According to A.4 - A.5), two parameters define the setting of the algorithm,

- testing time scale $\lambda$, defined as $\lambda=\frac{\Delta t^{C}}{\Delta t^{P}}=\frac{\Delta t^{S}}{\Delta t^{N}}$

- subcycling $m$, defined as $m=\frac{\Delta t^{N}}{\Delta t^{P}}=\frac{\Delta t^{S}}{\Delta t^{C}}$

Time steps $\Delta t^{N}$ and $\Delta t^{P}$ refer to simulation time, which is the virtual time axis defined by the time integration process. As an example, the seismic ground motion history refers to the simulation time axis. In particular, $\Delta t^{N}$ is the time step adopted for integrating the NS response while $\Delta t^{P}$ refers to the PS. On the other hand, $\Delta t^{S}$ and $\Delta t^{C}$ refer to wall-clock time measured in the laboratory. The former, $\Delta t^{S}$, defines the maximum solving time allocated to compute the NS response while the latter, $\Delta t^{C}$, is the actuator controller time step, which typically ranges between 1 and $2 \mathrm{msec}$. In order to minimize discontinuity of actuator trajectories, the PS equation should be evaluated within a single controller time step $\Delta t^{C}$.

The testing time scale $\lambda$ defines the ratio between wall-clock and simulation time rates. In particular, $\lambda=1$ corresponds to real-time HS. If $\lambda>1$, the simulation time flow is slower than the wall-clock time. This case corresponds to the pseudodynamic HS regime. When the response of the PS is not rate-dependent, $\lambda$ usually ranges between 50 and 200. This approach reduces displacement tracking errors as well as the destabilizing effect of actuator delay, which typically ranges from 10 to $20 \mathrm{msec}$. The subcycling parameter $m$ allows for adjusting $\Delta t^{S}$, which is the fixed time window allocated for solving the NS response. Accordingly, optimal tuning of testing time scale $\lambda$ and subcycling $m$ is a trade-off between computational accuracy and experimental capacity. For a detailed description of the partitioned time integration algorithm, the reader should refer to [32]. 


\section{Acknowledgments}

This work was supported by the Swiss Secretariat of Education Research and Innovation (SERI) - Swiss Space Office (SSO) [THERMICS MdP2016 Project (Thermo-Mechanical Virtualization of Hybrid Flax/Carbon Fiber Composite for Spacecraft Structures), grant number REF-1131-61001].

\section{References}

\section{References}

[1] F. G. Shellenberg A.H., Mahin S.A., "Advanced Implementation of Hybrid Simuation," Tech. Rep. 104, Pacific Earthquake Engineering Research Center, 2009.

[2] O. S. Bursi, G. Abbiati, E. Cazzador, P. Pegon, and F. J. Molina, "Nonlinear heterogeneous dynamic substructuring and partitioned FETI time integration for the development of low-discrepancy simulation models," International Journal for Numerical Methods in Engineering, vol. 112, pp. 1253-1291, 112017.

[3] V. Terzic, B. Stojadinovic, and M. Asce, "Hybrid Simulation of Bridge Response to Three-Dimensional Earthquake Excitation Followed by Truck Load," Journal of Structural Engineering, vol. 140, no. 8, p. A4014010, 2014.

[4] R. Christenson, Y. Z. Lin, A. Emmons, and B. Bass, "Large-Scale Experimental Verification of Semiactive Control through Real-Time Hybrid Simulation," Journal of Structural Engineering, vol. 134, no. 4, pp. 522$534,2008$.

[5] T. Sauder, S. Marelli, K. Larsen, and A. J. Sørensen, "Active truncation of slender marine structures: Influence of the control system on fidelity," Applied Ocean Research, vol. 74, pp. 154-169, 52018.

[6] C. A. Whyte, K. R. Mackie, and B. Stojadinovic, "Hybrid Simulation of Thermomechanical Structural Response," Journal of Structural Engineering, vol, 142, p. 04015107, 22016.

[7] A. Franza and A. M. Marshall, "Centrifuge and Real-Time Hybrid Testing of Tunneling beneath Piles and Piled Buildings," Journal of Geotechnical and Geoenvironmental Engineering, no. 3, p. 04018110, 2018.

[8] M. Vasmel, J. O. A. Robertsson, D.-J. van Manen, and A. Curtis, "Immersive experimentation in a wave propagation laboratory," The Journal of the Acoustical Society of America, vol. 134, no. 6, pp. EL492EL498, 2013.

[9] T. S. Becker, D. J. Van Manen, C. M. Donahue, C. Bärlocher, N. Börsing, F. Broggini, T. Haag, J. O. Robertsson, D. R. Schmidt, S. A. Greenhalgh, and T. E. Blum, "Immersive Wave Propagation Experimentation: Physical Implementation and One-Dimensional Acoustic Results," Physical Review X, vol. 8, no. 3, p. $31011,2018$.

[10] B. A. Bradley, "A critical examination of seismic response uncertainty analysis in earthquake engineering," Earthquake Engineering \&5 Structural Dynamics, vol. 42, pp. 1717-1729, 92013.

[11] A. Saltelli, M. Ratto, T. Andres, F. Campolongo, J. Cariboni, D. Gatelli, M. Saisana, and S. Tarantola, Global Sensitivity Analysis. The Primer, vol. 76. Chichester, UK: John Wiley \& Sons, Ltd, 122007.

[12] B. Sudret, "Global sensitivity analysis using polynomial chaos expansions," Reliability Engineering \& System Safety, vol. 93, pp. 964-979, 72008.

[13] L. Le Gratiet, S. Marelli, and B. Sudret, "Metamodel-Based Sensitivity Analysis: Polynomial Chaos Expansions and Gaussian Processes," in Handbook of Uncertainty Quantification, pp. 1289-1325, Cham: Springer International Publishing, 2017.

[14] D. Xiu and G. E. Karniadakis, "The Wiener-Askey Polynomial Chaos for Stochastic Differential Equations," SIAM Journal on Scientific Computing, vol. 24, pp. 619-644, 12002.

[15] M. Berveiller, B. Sudret, and M. Lemaire, "Stochastic finite element: a non intrusive approach by regression," European Journal of Computational Mechanics, vol. 15, pp. 81-92, 12006. 
[16] G. Blatman and B. Sudret, "Adaptive sparse polynomial chaos expansion based on least angle regression," Journal of Computational Physics, vol. 230, pp. 2345-2367, 32011.

[17] B. Iooss and P. Lemaître, "A review on global sensitivity analysis methods," arXiv:1404.2405 [math, stat], Apr. 2014. arXiv: 1404.2405.

[18] D. Harenberg, S. Marelli, B. Sudret, and V. Winschel, "Uncertainty quantification and global sensitivity analysis for economic models," Quantitative Economics, vol. 10, no. 1, pp. 1-41, 2019.

[19] M. Knabenhans, J. Stadel, S. Marelli, D. Potter, R. Teyssier, L. Legrand, A. Schneider, B. Sudret, L. Blot, S. Awan, C. Burigana, C. S. Carvalho, H. Kurki-Suonio, and G. Sirri, "Euclid preparation: II. The EuclidEmulator - a tool to compute the cosmology dependence of the nonlinear matter power spectrum," Monthly Notices of the Royal Astronomical Society, vol. 484, pp. 5509-5529, Jan. 2019.

[20] M. Ahmadizadeh and G. Mosqueda, "Online energy-based error indicator for the assessment of numerical and experimental errors in a hybrid simulation," Eng. Struct., vol. 31, no. 9, pp. 1987-1996, 2009.

[21] G. Abbiati, V. La Salandra, O. S. Bursi, and L. Caracoglia, "A composite experimental dynamic substructuring method based on partitioned algorithms and localized Lagrange multipliers," Mechanical Systems and Signal Processing, vol. 100, pp. 85-112, 22018.

[22] I. M. Sobol', "Sensitivity Estimates for Nonlinear Mathematical Models," tech. rep., 1993.

[23] E. Torre, S. Marelli, P. Embrechts, and B. Sudret, "Data-driven polynomial chaos expansion for machine learning regression," Journal of Computational Physics, vol. 388, pp. 601-623, 72019.

[24] G. Deman, K. Konakli, B. Sudret, J. Kerrou, P. Perrochet, and H. Benabderrahmane, "Using sparse polynomial chaos expansions for the global sensitivity analysis of groundwater lifetime expectancy in a multi-layered hydrogeological model," Reliab. Eng. Sys. Safety, vol. 147, pp. 156-169, 2016.

[25] S. Marelli and B. Sudret, "UQLab: A Framework for Uncertainty Quantification in Matlab," in Vulnerability, Uncertainty, and Risk, pp. 2554-2563, American Society of Civil Engineers, 62014.

[26] S. Marelli and B. Sudret, "UQLab user manual - Polynomial chaos expansions," tech. rep., Chair of Risk, Safety \& Uncertainty Quantification, ETH Zurich, 2019. Report \# UQLab-V1.2-104.

[27] S. Marelli, C. Lamas, K. Konakli, C. Mylonas, P. Wiederkehr, and B. Sudret, "UQLab user manual Sensitivity analysis," tech. rep., Chair of Risk, Safety \& Uncertainty Quantification, ETH Zurich, 2019. Report \# UQLab-V1.2-106.

[28] M. Dabaghi and A. Der Kiureghian, "Simulation of orthogonal horizontal components of near-fault ground motion for specified earthquake source and site characteristics," Earthquake Engineering 85 Structural Dynamics, vol. 47, pp. 1369-1393, 52018.

[29] G. Abbiati, H. Vincent, R. Julien, and S. Bozidar, "Thermo-mechanical virtualization of hybrid flax/carbon fiber composite for spacecraft structures. thermics project, final report.," 2018.

[30] S. Dubreuil, M. Berveiller, F. Petitjean, and M. Salaün, "Construction of bootstrap confidence intervals on sensitivity indices computed by polynomial chaos expansion," Reliability Engineering 85 System Safety, vol. 121, pp. 263-275, 2014.

[31] M. Brun, A. Batti, A. Combescure, and A. Gravouil, "External coupling software based on macro- and micro-time scales for explicit/implicit multi-time-step co-computations in structural dynamics," Finite Elements in Analysis and Design, vol. 86, pp. 101-119, 92014.

[32] G. Abbiati, I. Lanese, E. Cazzador, O. S. Bursi, and A. Pavese, "A computational framework for fast-time hybrid simulation based on partitioned time integration and state-space modeling," Structural Control and Health Monitoring, pp. 1-28, 72019. 